\title{
Transatlantica
}

Revue d'études américaines. American Studies Journal

\section{Représentation, simulacre et identité dans l'œuvre de Cindy Sherman}

"Cindy Sherman » (Musée du Jeu de Paume - Site Concorde, 16 mai $2006-3$ septembre 2006)

\section{Laetitia Barrière}

\section{(2) OpenEdition}

Journals

Édition électronique

URL : http://journals.openedition.org/transatlantica/1169

DOI : 10.4000/transatlantica. 1169

ISSN : $1765-2766$

Éditeur

AFEA

Référence électronique

Laetitia Barrière, « Représentation, simulacre et identité dans l'œuvre de Cindy Sherman »,

Transatlantica [En ligne], 2 | 2006, mis en ligne le 17 janvier 2007, consulté le 29 avril 2021. URL : http:// journals.openedition.org/transatlantica/1169; DOI : https://doi.org/10.4000/transatlantica.1169

Ce document a été généré automatiquement le 29 avril 2021.

\section{cc)}

Transatlantica - Revue d'études américaines est mis à disposition selon les termes de la licence Creative Commons Attribution - Pas d'Utilisation Commerciale - Pas de Modification 4.0 International. 


\title{
Représentation, simulacre et identité dans l'œuvre de Cindy Sherman
}

\author{
"Cindy Sherman » (Musée du Jeu de Paume - Site Concorde, 16 mai \\ $2006-3$ septembre 2006)
}

\section{Laetitia Barrière}

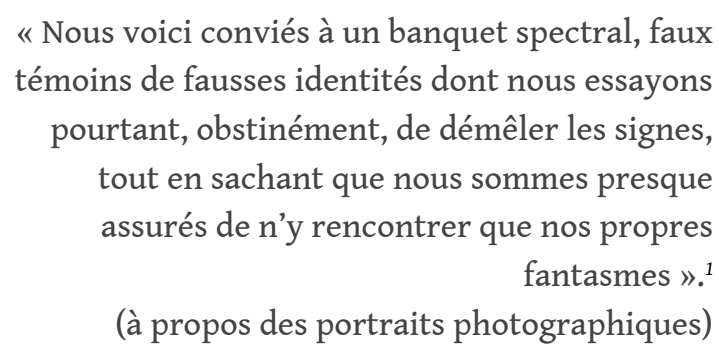

Les notions d'identité et de photographie sont étroitement liées comme en témoigne le concept même de "photo d'identité ». C'est autour de ce thème central que fut organisée l'exposition "Cindy Sherman », au Musée du Jeu de Paume - Site Concorde, du 16 mai 2006 au 3 septembre 2006.

2 La dernière exposition consacrée à Sherman en France remonte au printemps 1999, "Cindy Sherman» au Capc-Musée d'art contemporain de Bordeaux. Celle du Jeu de Paume remet à jour son œuvre, offrant aux spectateurs la possibilité de découvrir ses derniers clichés. Conçue avec Sherman elle-même, l'exposition relève d'une rétrospective davantage que d'une simple exposition. En effet, ses photos y sont présentées par ordre chronologique : les spectateurs abordent ainsi chaque mouvement de son parcours de manière distincte, tout en suivant, à travers les différentes salles de l'exposition, l'évolution globale de son travail.

3 A la fois modèle et photographe, Sherman expose son propre corps, modelé à l'infini, comme base invariante de ses photographies. Support de diverses manipulations et métamorphoses, il est à la base de son entreprise photographique. Il en résulte un ensemble de prises de vues qui sont autant de facettes de la photographe qui la 
représentent sans pour autant la dévoiler. Toutefois, ce qui nous est donné à voir se situe aux antipodes de l'autoportrait. Les propos de Sherman sur son travail préparatoire aux prises photographiques en témoignent : dans de nombreux entretiens, Sherman explique ainsi que lorsqu'elle aborde cette phase préparatoire, elle se sert d'un miroir placé à côté de l'objectif de son appareil et que c'est au moment où elle ne se reconnaît plus dans le miroir qu'elle prend le cliché.

4 La notion du corps comme image et comme support est illustrée par le court métrage projeté dans la première salle de l'exposition où Sherman figure comme un corps de poupée en deux dimensions, découpé dans du papier, nu, manipulé par des mains anonymes, assorti d'une panoplie de vêtements de toutes sortes destinés à modifier son apparence.

5 Les identités féminines multiples révélées par ces images visent à mettre en évidence les conventions sociales et culturelles qui ont dérobé aux femmes leur individualité propre : désormais, elles doivent se conformer à une norme. Modelée par les médias, inculquée par la culture, cette norme lie étroitement la femme à un certain nombre d'attributs dits "féminins»-fragilité, séduction, mystère (pour n'en citer que quelques-uns) auxquels les femmes doivent se conformer pour exister. C'est à ce carcan du canon et de l'idéal que Sherman s'attaque en premier lieu, multipliant les identités pour produire un kaléidoscope d'images qui questionne la notion même de modèle.

6 Toute photographie, aussi vraisemblable soit-elle, n'étant jamais, par essence, qu'une représentation, Sherman pousse cette définition à l'extrême. Création, tromperie et artifice : son œuvre est mise en scène, utilisant toutes les ressources du medium pour faire de la photographie une affaire de fiction. Des Untitled A-E de 1975 aux Clowns de 2003-2004, le fil conducteur de cette rétrospective est le simulacre, son thème de prédilection, jusqu'à l'obsession. Au fil du temps, la norme devient monstre tandis que la fantaisie bascule vers la folie.

$7 \mathrm{Au}$ début de sa carrière, Sherman s'intéresse aux médias qui, selon elle, ont façonné l'image des femmes du vingtième siècle. Elle en retient les principaux archétypes qu'elle subvertit, en travaillant certains clichés propres au cinéma, à la télévision ou au monde des magazines. Parmi ses premières photographies en noir et blanc figure la série des Untitled Film Stills de 1977, dans laquelle elle incarne des protagonistes du cinéma des années 50 et des séries $B$ de la télévision. Sherman s'approprie ces stéréotypes pour les critiquer et nous faire remettre en cause leur validité. Untitled Film Still 34 présente la femme tentatrice par excellence: allongée dans un lit aux draps froissés, dans une pose langoureuse, elle porte une chemise largement ouverte sur son décolleté. Un autre archétype présenté est celui de la femme fragile, en pleurs, de Untitled Film Still 27, dont le désarroi est intensifié par son visage baigné de larmes et par les traces noires du maquillage qui a coulé sur les joues. Dans un cas comme dans l'autre, tous les marqueurs sont là pour souligner le cliché que constitue chaque situation et le prendre à contre-pied. Ces femmes souvent idéalisées ne sont plus des modèles vers lesquels tendre, mais au contraire des personnages creux. Racoleuse, star de cinéma, ménagère, coquette, garçon manqué, Sherman crée un répertoire d'identités, d'une étonnante diversité, mais, alors même qu'elle les crée, elle semble les désavouer en forçant le trait de la représentation : si les photos de Sherman ne sont que des créations de toutes pièces, alors peut-être ne sommes-nous aussi que des produits entièrement fabriqués par les médias qui façonnent notre identité selon des codes et 
des modes pré-établis. Littéralement prisonnières des clichés qu'elles incarnent, ces femmes sont condamnées à n'être que des objets de série.

C'est Sherman elle-même qui incarne ces différentes femmes, se prêtant au jeu de la dissémination, pour représenter la notion même d'unité perdue. Abandonnées, fragiles, vulnérables, sans échappatoire, les femmes captives qu'elle met en scène se situent en position de subordination par rapport au regard, dont on peut supposer, au vu des poses choisies pour le retenir, qu'il est masculin. Le sujet de la photographie, qui devient l'objet du regard, semble dépourvu de tout pouvoir, sous l'emprise de l'autre. A l'instar du négatif photographique, qui inverse le clair et le sombre, il faut voir l'envers du décor que ses clichés désignent. Bien des photographies de cette période ne portent pas de titre, signe du désir de l'artiste de n'imposer aucun cadre narratif prédéfini à ses images, comme pour laisser à chacun la possibilité d'en définir le sens. Mais, en dépit de ce refus d'un cadrage trop net, ces photos font référence à un ensemble de codes attestés que le spectateur reconnaît, d'une manière plus ou moins précise, et qui guide son appréciation.

9 La multiplication des représentations constitue en soi le signe que la machine tourne à vide et que tout est modèle de consommation et de reproduction. Privés de traits spécifiques, les personnages de ses Untitled Film Stills perdent leur identité propre, de sorte que chacun peut tour à tour s'identifier à ce qui est représenté. Sherman opère ainsi ce renversement qui consiste à nier le sujet pour que chacun puisse devenir objet.

Le processus de réification des femmes se poursuit dans les photos en couleur des années 80 , en particulier dans les Centerfolds/Horizontals. Cette série, faite sur commande pour le compte de la revue Artforum, s'inspire des "revues de charme », un genre dont Sherman reprend les codes pour les subvertir par le biais de divers moyens : outre l'éclairage intense et la vue en plongée qui compresse les corps, les postures figées et les décors domestiques n'invitent pas à la moindre tentation. Sherman y exprime ainsi ses revendications féministes : loin de subjuguer, ses personnages sont soumis et le regard du spectateur devient voyeur, ce qui permet de dénoncer le propos dont la femme est l'objet.

11 Par le biais de remaniements successifs de la représentation, Sherman se ressaisit ainsi de la notion d'identité. Fashion Shots s'inscrit dans une nouvelle démarche de dénonciation vigoureuse des canons de la mode. Aux antipodes des corps lisses et parfaits qui défilent habituellement dans ce domaine, Sherman montre ce qu'il est convenu d'appeler les « victimes de la mode ». C'est notamment ce qu'illustre un cliché où figure une femme qui porte les vêtements d'un créateur et dont les cheveux ébouriffés cachent le visage: s'avançant les poings crispés, la furie provoque plus l'épouvante que l'envie.

12 Progressivement, Sherman s'éloigne des conventions, même détournées. Ses sujets deviennent étranges, surprenants, voire monstrueux ; les repères culturels qui balisent notre vision du monde sont bouleversés. Cette mise à distance est signifiée par la présence, dans ses photographies plus tardives, de poupées et de mannequins, et l'usage particulier qu'elle en fait. Tout d'abord, dans les scènes de contes de fées horrifiques (sobrement intitulés Fairy Tales), son utilisation de figurines traditionnellement associées aux petites filles, vient renforcer la déconstruction des rôles à laquelle se livre son œuvre. Sherman joue à la poupée, mais à sa façon, en les faisant entrer dans un monde d'abomination. Les poupons deviennent des monstres comme dans Untitled Film Still 187, où le personnage est composé d'une effrayante figure 
de clown, d'un ventre et de seins énormes, d'un nez à la place du nombril et de deux pieds minuscules par rapport au reste du corps. La monstruosité est accentuée par les proportions inhabituelles du personnage qui sont elles-mêmes renforcées par la prise de vue en contre-plongée. C'est le même principe de difformité qui domine dans une photographie reproduite dans l'ouvrage intitulé Cindy Sherman-Retrospective ${ }^{2}$ où le corps de l'enfant est créé par des parties juxtaposées les unes aux autres sans être assemblées, formant donc un tout hideux. Par ailleurs, outre son caractère informe, l'enfant tient dans sa main droite un glaive aux dimensions disproportionnées et sa tête arrachée est disposée sur ce qui ressemble à un calice. Celui-ci fait écho au Saint-Graal qui donne la vie, nourrit et éclaire. La table recouverte d'un drap rouge sang sur laquelle ce calice est posé est celle du sacrifice, incarné ici par le corps dépecé. A l'instar de la quête du Saint-Graal qui est entravée par l'aveuglement des hommes, Sherman souligne ici l'entrave du désir et le matérialisme dans la quête d'identité des individus, et suggère une délivrance par la séparation de ce corps qui nous enferme dans un carcan. De plus, la tête du poupon posée dans un coin rappelle les Vanités: Sherman avertit de la futilité du désir et de la fragilité de la vie face à notre mort inéluctable.

L'ajout d'accessoires qui sont mis en évidence indique que ces derniers peuvent être enlevés et sont ainsi autant de masques, prêts à tomber. Ces éléments supplémentaires renforcent le caractère fictionnel, et même théâtral, de la photographie de Cindy Sherman. L'horreur en arrive à basculer vers la dérision. Les portraits, qui ne sont qu'illusion, se vident de sens : puisque les personnages ne sont que représentations ou copies, l'original s'efface. Par cette dissociation entre l'image et le réel, un espace intermédiaire est créé, où se perd l'identité du sujet. De manière paradoxale, au lieu d'affirmer l'objet photographié, Sherman le déconstruit par le biais d'une dissémination qui est à l'origine d'une puissante perplexité.

Par ailleurs, Sherman utilise des mannequins, qui érigent un corps normé, sans signe distinctif, en standard. Le remplacement d'un personnage en chair et en os par des pastiches en plastique vise à dénoncer, par de nouveaux moyens, l'aspect factice de l'identité. Cet élément artificiel stigmatise le rôle imposé aux femmes qui dénature leur identité réelle. La répugnance s'installe déjà avec Untitled 263, qui fait écho au tableau de Courbet L'Origine du monde, et qui présente un corps hybride composé de deux basventres, un masculin et un féminin, reliés au niveau de l'abdomen, c'est-à-dire sans tronc. Les têtes de chacun des corps sont présentes dans la photographie, coupées et posées dans deux coins, comme pour exprimer une forme de conscience de la dislocation du sujet. Egalement amputé de sa partie inférieure, le corps a les jambes coupées au niveau du haut des cuisses. Sherman attire l'attention sur le sexe des personnages par un élément qui surprend, notamment l'anneau sur le pénis et le cordon de tampon qui sort du sexe féminin. Ce n'est pas le corps féminin comme pouvant donner la vie qui est exhibé: ces parties génitales évoquent plus la pornographie que l'acte d'amour. Le bandeau joliment noué qui unit les deux basventres contraste avec la bestialité crue de la scène. Dans un esprit voisin, Untitled 250 dévoile une femme nue sur le dos. Malgré sa pose naturelle, allongée, les bras derrière la tête, elle n'a ni jambe, ni dos, et sa figure est celle d'une personne âgée, comme en témoignent son visage ridé et ses cheveux gris. Peu à peu mutilés, démembrés et déconstruits, ces corps amputés ou défaits expriment la désagrégation de l'identité. 
Déjà largement mise à mal, l'identité est dissoute dans les scènes de mort de la série suivante, dans laquelle Sherman s'écarte du normal et dévie vers l'extra-ordinaire, jusqu'au grotesque. Des scènes excentriques et morbides prennent le pas sur celles du quotidien. Les femmes fatales laissent place aux monstres, puis à la mort, aux déchets. Les normes du beau, du propre, de l'idéal féminin sont ébranlées au point de céder ; l'effroi s'intensifie jusqu'à provoquer la répugnance dans Disasters, une série de photos dans laquelle le corps commence par passer à l'arrière-plan, pour ensuite disparaître. Il n'y a plus d'enveloppe charnelle sur les clichés : dans Untitled 175, le corps de femme est tout juste évoqué par son reflet dans les lunettes de soleil, alors que des objets inanimés occupent le centre de la photo. Dans d'autres clichés, le corps finit par disparaître, laissant place au spectacle de sa décomposition, dans le sens physique du terme, exposant ses matières (vomissements et moisissures). La dichotomie intérieur/ extérieur tombe et le spectateur est confronté avec ce qu'il ne veut pas voir. Le monde n'est plus l'univers familier que le spectateur connait ; il a été remplacé par un espace chaotique et répugnant. Les morceaux d'humains disséminés sur les clichés sont autant de pièces d'un puzzle qui ne peuvent être assemblées, signe d'un moi qui n'est plus une entité cohérente. De manière paradoxale, c'est dans cette négation que l'identité du sujet s'affirme.

16 A mesure que l'œuvre de Sherman progresse vers la marginalité, la frontière entre le noble et le vulgaire disparaît tandis que la distinction entre le beau et le laid s'efface. Ses photos deviennent hybrides, mêlant un contenu qui inspire l'effroi et la recherche d'une certaine forme esthétique. Untitled 19 donne en gros plan, sous de longs cheveux blonds, un téton, une main manucurée et un visage tronqué, infesté d'insectes, ce qui laisse supposer son état de décomposition. L'éclat des couleurs (bleu de l'œil révulsé, blanc de l'œil et paupière bleuie) et la perfection du dessin des sourcils contrastent avec la saleté ostentatoire du front. La netteté des contours s'oppose au chaos de la dégradation et la beauté contraste en tous points avec l'horreur. Face à l'inconfort ou même l'effroi qu'inspirent ces clichés, le spectateur a le recours d'une lecture qui démonte les rouages de la mise en scène, selon un mécanisme suggéré et accentué tout au long du parcours de la photographe.

Pour subvertir de nouveaux champs de la représentation, Sherman se tourne enfin vers des classiques de l'histoire de l'art qu'elle parodie dans les History Portraits par le biais de doubles grotesques, prenant encore une fois la tradition à contre-pied. Optant une nouvelle fois pour une approche où la transgression est de mise, Sherman s'approprie les toiles des maîtres par le biais du détournement. Face à ces History Portraits, il nous semble en effet reconnaître des répliques de toiles originales, sans qu'il en soit toujours possible de pointer du doigt l'original. Excepté trois d'entre eux parodiant respectivement le «Bacchus Malade» de Caravage, «La Venus de Melun» de Jean Fouquet et «La Fornarina » de Raphaël, les autres photographies sont construites au moyen de morceaux venus de divers tableaux. Il en résulte des portraits hybrides, les traits grossis à l'extrême, jouant sur le registre de l'art noble des portraits appartenant à la peinture d'histoire, ainsi que sur le laid, le trivial. Dans ces séries, les prothèses en tous genres sont utilisées, exhibées comme telles, reformulant ainsi, d'une manière voyante, la question de l'identité. Que dire en effet de la difformité des personnages de Untitled 213 (nez et sourcils démesurés) ou du nez pointu et disgracieux de Untitled 211 ? L'œuvre de Sherman persiste à confronter le spectateur à la notion d'artifice, par le biais de contenus renouvelés et de moyens techniques variés. Dans les History Portraits, 
le caractère théâtral des scènes, marqué par la présence de rideaux, accuse le caractère de représentation de la série.

Pour le tableau inspiré de "La Fornarina », il est important de souligner que Sherman n'a pas travaillé à partir du tableau original mais qu'elle s'est fondée sur une reproduction. On note ainsi un effet de mise en abîme réalisé par cette photographie qui n'est finalement qu'une copie déformante d'une copie d'une œuvre de Raphaël. Ici, la photographie devient un écho parodique de la peinture, la peinture devient photographie, et la femme photographe du vingtième siècle se substitue à l'homme peintre des siècles passés, illustrant ainsi la reprise du pouvoir par les femmes dans le domaine de l'art.

L'exposition se termine sur la série des Clowns, clichés pris en numérique, qui ont ainsi pu être retouchés. Sherman ajoute en arrière-plan des motifs bariolés et nous présente non plus un seul mais plusieurs personnages par photographie. Face à de tels clichés, le spectateur est confronté à une profusion de couleurs plus qu'à la terreur singulière des œuvres précédentes. Cette figure du clown, qui ouvre l'exposition et sur laquelle elle se termine, établit un fil directeur entre les différentes étapes du parcours, désignant la mascarade de la production de Sherman. Jouant sur les registres du burlesque et/ou du grotesque, le clown déplace l'ambivalence propre à la photographie de Sherman sur le terrain du tragique et du comique. C'est dans cet écart que Sherman loge l'identité de ses personnages.

Si l'on en croit les nombreux masques dans ses clichés, rien n'est ce qu'il laisse paraître, et c'est dans l'écart que désigne son œuvre que se loge la problématique de l'identité que travaille son œuvre. De manière paradoxale, il semble que, plus Sherman s'exhibe dans ses photographies, plus elle s'échappe en devenant autre. Grimée, Sherman est méconnaissable à tel point que lorsqu'on la voit au naturel pour la première fois, il est difficile de la reconnaitre alors que nos yeux l'ont vu figurer sur des dizaines de tirages. $\mathrm{Au}$ bout du compte, plus elle se cache derrière ses portraits, plus elle se révèle en tant qu'artiste.

Par son œuvre polymorphe, Sherman remet en cause non seulement les codes féminins en vogue à la fin du vingtième siècle aux Etats-Unis, mais, au-delà, elle pose des questions fondamentales à l'art en général, utilisant parfois le mode de la provocation pour redéfinir des concepts-clés tels que ceux de la beauté et de la laideur, la surface et la profondeur. Par ce décloisonnement permanent entre le réel et sa représentation, Sherman crée un espace intermédiaire où se perd et s'élabore l'identité du sujet.

\section{NOTES}

1.Durand, Régis. La part de l'ombre - essais sur l'expérience photographique. Paris : Editions La Différence, 1990, p. 56.

2. Cindy Sherman - Retrospective. Thames \& Hudson, 1998, p. 171 
INDEX

Thèmes : Trans'Arts 